\title{
Accommodating Sensor Bias in MRAC for State Tracking
}

\author{
Parag Patre* and Suresh M. Joshi ${ }^{\dagger}$
}

\begin{abstract}
The problem of accommodating unknown sensor bias is considered in a direct model reference adaptive control (MRAC) setting for state tracking using state feedback. Sensor faults can occur during operation, and if the biased state measurements are directly used with a standard MRAC control law, neither closed-loop signal boundedness, nor asymptotic tracking can be guaranteed and the resulting tracking errors may be unbounded or unacceptably large. A modified MRAC law is proposed, which combines a bias estimator with control gain adaptation, and it is shown that signal boundedness can be accomplished, although the tracking error may not go to zero. Further, for the case wherein an asymptotically stable sensor bias estimator is available, an MRAC control law is proposed to accomplish asymptotic tracking and signal boundedness. Such a sensor bias estimator can be designed if additional sensor measurements are available, as illustrated for the case wherein bias is present in the rate gyro and airspeed measurements. Numerical example results are presented to illustrate each of the schemes.
\end{abstract}

Keywords: Sensor fault accommodation, adaptive control, sensor bias.

\section{Introduction}

Control of uncertain systems in the presence of actuator and sensor faults is an important and challenging problem. Two traditional approaches to this problem are fault detection, identification (FDI)-based control reconfiguration, and direct adaptive control. Although considerable research effort has focused on actuator fault accommodation in the past, literature addressing sensor fault accommodation appears to be rather limited. However, sensor faults have been determined to be a cause in several aircraft accidents (e.g., radio altimeter fault, ${ }^{1}$ angle-of-attack sensor fault ${ }^{2}$ airspeed sensor fault $\left.{ }^{3}\right)$.

Direct model reference adaptive control (MRAC) methods aim to control uncertain systems using controller gains that are adaptively adjusted to achieve a performance close to that of a reference model while maintaining system stability and close tracking of the reference model response. Direct MRAC has been known to be an effective method for state- or output- tracking. Direct MRAC schemes that use state feedback for state-tracking (SFST) have the advantage of simplicity of implementation while achieving effective state tracking in the presence of parameter uncertainties. ${ }^{4}$ Such schemes have also been extended to the case with actuator failures, ${ }^{5},{ }^{6} .{ }^{7}$ However, aside from actuator faults, sensor faults may corrupt the measurements. Any sensor bias prior to the operation can be compensated for by appropriate off-line calibration methods. However, unknown biases can appear during operation in sensors such as rate gyros, accelerometers, altimeter, etc. The most common approach to deal with such sensor faults is to use redundant sensor packages. However, common-mode failures can occur across all the sensors, and moreover, each sensor may develop a different unknown bias. If used directly in a MRAC control law, such offsets in sensor measurements can have detrimental effects on closed-loop stability.

Sensor bias estimation and accommodation has been a topic of considerable research. For example, bias estimation for multisensor systems has been investigated in, $, 8,{ }^{9} .{ }^{10}$ The problem of setpoint tracking with sensor bias was discussed in, ${ }^{11}$ and MRAC schemes for output feedback for output tracking in the presence

\footnotetext{
*NASA Postdoctoral Program Fellow, Mail Stop 308 NASA Langley Research Center, Hampton, VA 23681; Email parag.patre@nasa.gov

†Senior Scientist for Control Theory, Mail Stop 308, NASA Langley Research Center, Hampton, VA 23681; Email suresh.m.joshi@nasa.gov; AIAA Fellow.
} 
of sensor faults have been developed (e.g., ${ }^{1213}$ ). However, accommodating sensor bias in SFST-MRAC has not been investigated to the knowledge of the authors.

In this paper, it is assumed that all the a priori bias has been compensated for by off-line calibration methods, and trim values of the system states are available at the start of the control operation. Then, direct MRAC is considered in the presence of any constant unknown sensor bias that may appear during the operation. If biased sensor signals are used directly in the MRAC law, signal boundedness and tracking cannot be proved, as discussed in Section II. A logical extension of the standard MRAC is proposed in Section III, which incorporates sensor bias estimation in the MRAC law, and shows that signal boundedness and bounded tracking error can be accomplished. To compensate for the effect of bias, an additional adaptive gain is included in this control law. The case wherein a sensor bias estimator is available is considered in Section IV. If the sensor bias estimator has asymptotic convergence properties, a modified MRAC law can be developed, which can guarantee signal boundedness and asymptotic tracking. As an example, Section V considers a longitudinal aircraft dynamics model with constant biases in the pitch-rate measurement gyroscope and in the airspeed measurement. Based on the availability of reliable pitch angle and position measurements, non-model-based observers are constructed and used in conjunction with adaptive control. The resulting MRAC scheme is shown to provide asymptotic tracking and signal boundedness. Section VI contains application examples of the proposed schemes, and concluding remarks are given in Section VII.

\section{Sensor Bias and Standard MRAC}

Consider a linear time-invariant plant described by:

$$
\begin{aligned}
\dot{x}(t) & =A x(t)+B u(t) \\
y(t) & =x(t)+\beta
\end{aligned}
$$

where $A \in R^{n \times n}$ is the system matrix assumed to be unknown, $B \in R^{n \times m}$ is a known input matrix, $x(t) \in R^{n}$ is the system state, and $u(t) \in R^{m}$ is the control input. $y(t) \in R^{n}$ is the available state measurement with an unknown constant bias $\beta \in R^{n}$.

The objective is to design an adaptive feedback control law using the available measurement $y(t)$ with unknown bias $\beta$, such that closed-loop signal boundedness is ensured and the system state $x(t)$ tracks the state of a reference model described by

$$
\dot{x}_{m}(t)=A_{m} x_{m}(t)+B_{m} r(t)
$$

where $x_{m} \in R^{n}$ is the reference model state, $A_{m} \in R^{n \times n}, B_{m} \in R^{n \times m}$, and $r(t) \in R^{m_{r}}\left(1 \leq m_{r} \leq m\right)$ is a bounded reference input used in system operation (e.g., pilot input in the case of aircraft).

It is assumed that the system $(A, B)$ and the reference model $\left(A_{m}, B_{m}\right)$ satisfy the SFST matching conditions, i.e., there exist gains $K_{1} \in R^{n \times m}, K_{2} \in R^{m \times m_{r}}$ such that

$$
A_{m}=A+B K_{1}^{T} ; B_{m}=B K_{2} \text {. }
$$

The reference model is customarily designed to capture the desired closed-loop response of the plant. For example, the reference model may be designed using optimal and robust control methods such as LQR, $H_{2}$, or $H_{\infty}$ methods. For the adaptive control scheme, only $A_{m}$ and $B$ need to be known. Moreover, there exist positive definite matrices $P=P^{T}, Q=Q^{T} \in R^{n \times n}$, such that the following Lyapunov inequality is satisfied:

$$
A_{m}^{T} P+P A_{m}<-Q \text {. }
$$

The standard MRAC SFST control law is given by

$$
u=\hat{K}_{1}^{T} y+\hat{K}_{2} r
$$

where $\hat{K}_{1}(t) \in R^{n \times m}, \hat{K}_{2}(t) \in R^{m \times m_{r}}$ are the adaptive gains. Substituting (5) into (1), the closed-loop plant is given by:

$$
\begin{aligned}
\dot{x} & =A x+B\left(\hat{K}_{1}^{T} y+\hat{K}_{2} r\right) \\
& =A x+B\left(K_{1}^{T} y+K_{2} r\right)+B\left(\tilde{K}_{1}^{T} y+\tilde{K}_{2} r\right) \\
& =\left(A+B K_{1}^{T}\right) x+B\left(\tilde{K}_{1}^{T} y+\tilde{K}_{2} r\right)+B K_{2} r+B K_{1}^{T} \beta
\end{aligned}
$$


where the fact that $y=x+\beta$ was used, and $\tilde{K}_{1}=\hat{K}_{1}-K_{1} ; \tilde{K}_{2}=\hat{K}_{2}-K_{2}$. Using the matching conditions (3) in (6), we get

$$
\dot{x}=A_{m} x+B_{m} r+B\left(\tilde{K}_{1}^{T} y+\tilde{K}_{2} r\right)+B K_{1}^{T} \beta .
$$

The last term in (7) represents a bias disturbance term, which will need to be compensated for by the adaptive control law. Defining the tracking error $e=x-x_{m}$, and using (2) and (7), we have

$$
\dot{e}=A_{m} e+B\left(\tilde{K}_{1}^{T} y+\tilde{K}_{2} r\right)+B K_{1}^{T} \beta .
$$

Due the presence of unknown sensor bias, $x(t)$ cannot be measured; therefore the tracking error $e(t)$ is not available. Thus, the standard MRAC control law in (5) cannot guarantee signal boundedness (including boundedness of the tracking error) in the presence of sensor bias in state feedback. The next section proposes a modified adaptive control law that aims to estimate and compensate for the sensor bias.

\section{Modified MRAC with Sensor Bias Estimation}

In this section, a bias estimator is proposed as a part of the adaptive control law that uses the available (biased) measurements, and can provide bounded state tracking error along with signal boundedness.

Let $\hat{\beta}(t)$ denote an estimate of the unknown sensor bias $\beta$. Using $\hat{\beta}$, define the 'corrected' state $\bar{x}(t) \in R^{n}$ as

$$
\bar{x}=y-\hat{\beta} .
$$

Therefore,

$$
\bar{x}=x+\beta-\hat{\beta}=x+\tilde{\beta} .
$$

where $\tilde{\beta}=\beta-\hat{\beta}$. Design an adaptive control law as

$$
u=\hat{K}_{1}^{T} y+\hat{K}_{2} r+\hat{k}_{3}
$$

where $\hat{K}_{1}(t) \in R^{n \times m}, \hat{K}_{2}(t) \in R^{m \times m_{r}}$, and $\hat{k}_{3}(t) \in R^{m}$ are the adaptive gains. Therefore, the closed-loop corrected-state equation is

$$
\begin{aligned}
\dot{\bar{x}} & =A x+B\left(\hat{K}_{1}^{T} y+\hat{K}_{2} r+\hat{k}_{3}\right)+\dot{\tilde{\beta}} \\
& =A x+B\left(K_{1}^{T} y+K_{2} r+k_{3}\right)+B\left(\tilde{K}_{1}^{T} y+\tilde{K}_{2} r+\tilde{k}_{3}\right)+\dot{\tilde{\beta}} \\
& =\left(A+B K_{1}^{T}\right) x+B\left(\tilde{K}_{1}^{T} y+\tilde{K}_{2} r+\tilde{k}_{3}\right)+B K_{2} r+B K_{1}^{T} \beta+B k_{3}+\dot{\tilde{\beta}}
\end{aligned}
$$

where $\tilde{K}_{1}=\hat{K}_{1}-K_{1}, \tilde{K}_{2}=\hat{K}_{2}-K_{2}$, and $\tilde{k}_{3}=\hat{k}_{3}-k_{3}$. The following matching conditions are assumed to be satisfied for some ideal gains $K_{1}, K_{2}, k_{3}$ :

$$
A_{m}=A+B K_{1}^{T}, B_{m}=B K_{2}, B K_{1}^{T} \beta=-B k_{3} .
$$

Using (12) and (9) in (11), we get

$$
\dot{\bar{x}}=A_{m} \bar{x}+B_{m} r+B\left(\tilde{K}_{1}^{T} y+\tilde{K}_{2} r+\tilde{k}_{3}\right)-A_{m} \tilde{\beta}+\dot{\tilde{\beta}} .
$$

Define a measurable auxiliary error signal $\hat{e}(t) \in R^{n}$ as

$$
\hat{e}=\bar{x}-x_{m} .
$$

Therefore, from (9), we have

$$
\hat{e}=x-x_{m}+\tilde{\beta}=e+\tilde{\beta} .
$$

Differentiating (14) with respect to time, the closed-loop auxiliary error system can be expressed as

$$
\dot{\hat{e}}=\dot{\bar{x}}-\dot{x}_{m} \text {. }
$$


Substituting (13) and (2) into (16) yields

$$
\dot{\hat{e}}=A_{m} \hat{e}+B\left(\tilde{K}_{1}^{T} y+\tilde{K}_{2} r+\tilde{k}_{3}\right)-A_{m} \tilde{\beta}+\dot{\tilde{\beta}} .
$$

The following theorem gives adaptive gain update and bias estimation laws that guarantee closed-loop signal boundedness as well as bounded tracking error.

Theorem 1: For the system given by (1), (2); the adaptive controller (10), the gain adaptation laws

$$
\begin{aligned}
\dot{\hat{K}}_{1} & =-\Gamma_{1} y \hat{e}^{T} P B \\
\dot{\hat{K}}_{2} & =-\Gamma_{2} B^{T} P \hat{e} r^{T} \\
\dot{\hat{k}}_{3} & =-\Gamma_{3} B^{T} P \hat{e}
\end{aligned}
$$

where $\Gamma_{1} \in R^{n \times n}, \Gamma_{2} \in R^{m \times m}, \Gamma_{3} \in R^{m \times m}$ are constant symmetric positive definite matrices and $P$ was defined in (4), and the bias estimation law

$$
\dot{\hat{\beta}}=-\eta P^{-1} A_{m}^{T} P \hat{e}
$$

where $\eta \in R$ is a tunable positive constant gain, guarantee that all the closed-loop signals including the adaptive gains and bias estimate are bounded and the tracking error $e(t)$ is bounded.

Proof: Define

$$
V=\hat{e}^{T} P \hat{e}+\sum_{i}^{n} \tilde{K}_{1 i}^{T} \Gamma_{1}^{-1} \tilde{K}_{1 i}+\sum_{i}^{m_{r}} \tilde{K}_{2 i}^{T} \Gamma_{2}^{-1} \tilde{K}_{2 i}+\tilde{k}_{3}^{T} \Gamma_{3}^{-1} \tilde{k}_{3}+\frac{1}{\eta} \tilde{\beta}^{T} P \tilde{\beta}
$$

where the subscript $i$ denotes the $i^{\text {th }}$ column of $\tilde{K}_{1}, \tilde{K}_{2}$. Differentiating (20) with respect to time, using (4), (17), properties of matrix trace, and the gain update laws in (18), the following expression is obtained upon simplification:

$$
\dot{V}<-\hat{e}^{T} Q \hat{e}-2 \hat{e}^{T} P A_{m} \tilde{\beta}-2 \hat{e}^{T} P \dot{\hat{\beta}}-\frac{2}{\eta} \tilde{\beta}^{T} P \dot{\hat{\beta}} .
$$

Using the bias estimation law of (19) in (21), we get

$$
\begin{aligned}
\dot{V} & <-\hat{e}^{T} Q \hat{e}-2 \hat{e}^{T} P A_{m} \tilde{\beta}+2 \eta \hat{e}^{T} A_{m}^{T} P \hat{e}+2 \tilde{\beta}^{T} A_{m}^{T} P \hat{e} \\
& =\quad-\hat{e}^{T} Q \hat{e}-\eta \hat{e}^{T} Q \hat{e}=-(1+\eta) \hat{e}^{T} Q \hat{e} .
\end{aligned}
$$

Therefore, $\dot{V}<0$, i.e., $V(T)$ is bounded for all $T$, and $\hat{e}(t), \hat{\beta}(t), y(t), \hat{K}_{1}, \hat{K}_{2}, \hat{k}_{3}$ are all bounded and $\hat{e}(t), \tilde{\beta}(t) \in L^{2}$. From (17), (19) and closed-loop signal boundedness, we have $\dot{\hat{e}}(t), \dot{\tilde{\beta}} \in L^{\infty}$, therefore $\lim _{t \rightarrow \infty} \hat{e}(t)=0$. Because $\hat{e}(t) \in L^{2} \cap L^{\infty}$ and $y(t) \in L^{\infty}, \dot{\hat{K}}_{1 i}, \dot{\hat{K}}_{2 i}, \dot{\hat{k}}_{3} \in L^{2} \cap L^{\infty}$. It can be verified that $\ddot{\hat{K}}_{1 i}, \ddot{\hat{K}}_{2 i}, \ddot{\hat{k}}_{3} \in L^{\infty}$, therefore, $\lim _{t \rightarrow \infty} \dot{\hat{K}}_{1 i}(t)=0, \lim _{t \rightarrow \infty} \dot{\hat{K}}_{2 i}(t)=0, \lim _{t \rightarrow \infty} \dot{\hat{k}}_{3}(t)=0$. That is, all signals and estimates are bounded, and $\lim _{t \rightarrow \infty}\left(\bar{x}-x_{m}\right)=0$.

If sufficient persistent excitation is present, $\tilde{\beta}(t)$ should approach 0 as $t \rightarrow \infty$, and therefore $e(t) \rightarrow 0$ as $t \rightarrow \infty$.

The adaptive control law in Theorem 1 guarantees stability (signal boundedness) and bounded tracking error. However, it would be desirable to achieve asymptotic tracking. In an effort to accomplish asymptotic tracking, the next section addresses the case when a separate asymptotically stable bias estimator is available (e.g., [14]).

\section{MRAC with Asymptotic Bias Estimation}

Suppose an asymptotically stable bias estimator is available. This section illustrates how such an estimator can be employed in a MRAC setting to achieve asymptotic state tracking.

Consider a bias estimator with estimation error dynamics of the form

$$
\dot{\tilde{\beta}}=A_{\beta} \tilde{\beta}
$$

where $\hat{\beta}(t)$ is an estimate of $\beta$, and

$$
\tilde{\beta}(t)=\beta-\hat{\beta}(t)
$$


is the estimation error. $A_{\beta} \in R^{n \times n}$ is a known asymptotically stable matrix, such that $\lim _{t \rightarrow \infty} \tilde{\beta}(t)=0$. Defining the adaptive control law as in (10) and proceeding as in Section III, we obtain (17). The following result gives an adaptive control law that guarantees signal boundedness and asymptotic tracking.

Theorem 2: For the system given by (1), (2); the adaptive controller (10), the bias estimator (22), and the gain adaptation laws

$$
\begin{aligned}
\dot{\hat{K}}_{1} & =-\Gamma_{1} y \hat{e}^{T} P B \\
\dot{\hat{K}}_{2} & =-\Gamma_{2} B^{T} P \hat{e} r^{T} \\
\dot{\hat{k}}_{3} & =-\Gamma_{3} B^{T} P \hat{e}
\end{aligned}
$$

where $\Gamma_{1} \in R^{n \times n}, \Gamma_{2} \in R^{m \times m}, \Gamma_{3} \in R^{m \times m}$ are constant symmetric positive definite matrices and $P$ was defined in (4), guarantee that all the closed-loop signals including adaptive gains are bounded and the tracking error $e(t) \rightarrow 0$ as $t \rightarrow \infty$.

Proof: Define

$$
V=\hat{e}^{T} P \hat{e}+\sum_{i}^{n} \tilde{K}_{1 i}^{T} \Gamma_{1}^{-1} \tilde{K}_{1 i}+\sum_{i}^{m_{r}} \tilde{K}_{2 i}^{T} \Gamma_{2}^{-1} \tilde{K}_{2 i}+\tilde{k}_{3}^{T} \Gamma_{3}^{-1} \tilde{k}_{3}+\tilde{\beta}^{T} P_{\beta} \tilde{\beta}
$$

where the subscript $i$ denotes the $i^{\text {th }}$ column of $\tilde{K}_{1}, \tilde{K}_{2}$; and $P_{\beta}=P_{\beta}^{T} \in R^{n \times n}$ is a positive definite solution to the Lyapunov inequality

$$
A_{\beta}^{T} P_{\beta}+P_{\beta} A_{\beta}<-Q_{\beta}
$$

for some positive definite matrix $Q_{\beta}=Q_{\beta}^{T} \in R^{n \times n}$. Differentiating (24) with respect to time, using (4), (17), (22), (25), and properties of matrix trace, the following expression is obtained upon simplification:

$$
\begin{aligned}
\dot{V} \leq & -\hat{e}^{T} Q \hat{e}-2 \hat{e}^{T} P\left(A_{m}-A_{\beta}\right) \tilde{\beta}-\tilde{\beta}^{T} Q_{\beta} \tilde{\beta}+2 \tilde{k}_{3}^{T}\left\{B^{T} P \hat{e}+\Gamma_{3}^{-1} \dot{\hat{k}}_{3}\right\} \\
& +2 \operatorname{Tr}\left[\tilde{K}_{1}^{T}\left\{y \hat{e}^{T} P B+\Gamma_{1}^{-1} \dot{\hat{K}}_{1}\right\}\right]+2 \operatorname{Tr}\left[\tilde{K}_{2}^{T}\left\{B^{T} P \hat{e} r^{T}+\Gamma_{2}^{-1} \dot{\hat{K}}_{2}\right\}\right] .
\end{aligned}
$$

Using the gain update laws (23) in (26), we get

$$
\begin{aligned}
\dot{V} & \leq-\hat{e}^{T} Q \hat{e}-2 \hat{e}^{T} P\left(A_{m}-A_{\beta}\right) \tilde{\beta}-\tilde{\beta}^{T} Q_{\beta} \tilde{\beta} \\
& =-z^{T} \bar{Q} z
\end{aligned}
$$

where $z \in R^{2 n}$ is defined as

$$
z=\left[\begin{array}{ll}
\hat{e} & \tilde{\beta}
\end{array}\right]^{T}
$$

and $\bar{Q} \in R^{2 n \times 2 n}$ is defined as

$$
\bar{Q}=\left[\begin{array}{cc}
Q & P\left(A_{m}-A_{\beta}\right) \\
\left(A_{m}-A_{\beta}\right)^{T} P & Q_{\beta}
\end{array}\right]
$$

Since $Q$ is positive definite, the matrix $\bar{Q}$ is positive definite iff

$$
Q_{\beta}-\left(A_{m}-A_{\beta}\right)^{T} P Q^{-1} P\left(A_{m}-A_{\beta}\right)>0 .
$$

$Q_{\beta}$ can be chosen such that $(27)$ is satisfied; therefore, $\dot{V}<0$, i.e., $V(T)$ is bounded for all $T$, and $\hat{e}(t), \hat{\beta}(t), y(t), \hat{K}_{1}, \hat{K}_{2}, \hat{k}_{3}$ are all bounded and $\hat{e}(t), \tilde{\beta}(t) \in L^{2}$. From (17), (22) and closed-loop signal boundedness, we have $\dot{\hat{e}}(t), \dot{\tilde{\beta}} \in L^{\infty}$, therefore $\lim _{t \rightarrow \infty} \hat{e}(t)=0$. From $(22), \lim _{t \rightarrow \infty} \tilde{\beta}(t)=0$. Therefore, from $(15), \lim _{t \rightarrow \infty} e(t)=0$. Because $\hat{e}(t) \in L^{2} \cap L^{\infty}$ and $y(t) \in L^{\infty}, \dot{\hat{K}}_{1 i}, \dot{\hat{K}}_{2 i}, \dot{\hat{k}}_{3} \in L^{2} \cap L^{\infty}$. It can be verified that $\ddot{\hat{K}}_{1 i}, \ddot{\hat{K}}_{2 i}, \ddot{\hat{k}}_{3} \in L^{\infty}$, therefore, $\lim _{t \rightarrow \infty} \dot{\hat{K}}_{1 i}(t)=0, \lim _{t \rightarrow \infty} \dot{\hat{K}}_{2 i}(t)=0, \lim _{t \rightarrow \infty} \dot{\hat{k}}_{3}(t)=0$. That is, all signals and estimates are bounded, and $\lim _{t \rightarrow \infty}\left(x-x_{m}\right)=0$. 


\section{Non-Model-Based Observers for Longitudinal Aircraft Dynamics}

In this section, we illustrate how sensor bias estimators can be developed and used in conjunction with MRAC. The bias estimates are an outcome of non-model-based observers, which additionally, also generate estimates of other measurable states. It is shown, how these additional state estimates, and the bias estimates are combined with MRAC to yield asymptotic state tracking even in the presence of unknown sensor bias. Specifically, we consider a fourth-order longitudinal dynamic model of an aircraft. The state variables are: true airspeed $v(t)$, angle-of-attack $\alpha(t)$, pitch angle $\theta(t)$, and pitch rate $q(t)$. Thus, the state is $x=$ $\left[\begin{array}{llll}v & \alpha & \theta & q\end{array}\right]^{T}$.

\section{V.A. Rate Gyro Bias Observer}

The pitch rate is usually measured by a rate gyro which is typically subject to bias buildup. Differentiating pitch angle to obtain the pitch rate is not advisable due to the presence of noise. Consider that the available state measurement $(y=x+\beta)$ has an unknown constant bias in the pitch rate measurement, such that

$$
\beta=\left[\begin{array}{llll}
0 & 0 & 0 & b
\end{array}\right]^{T}
$$

where $b$ is an unknown constant. Thus, the measured pitch rate $\left(q_{m}(t)\right)$ can be expressed as

$$
q_{m}=q+b=\dot{\theta}+b .
$$

Therefore, the actual pitch rate $(q=\dot{\theta})$ is

$$
\dot{\theta}=-b+q_{m} .
$$

The attitude (pitch angle) sensor usually consists of a two degrees-of-freedom (DOF) vertical gyro. The measurements degrade when the gimbal axes are not orthogonal, or a gimbal lock occurs when the spin axis is aligned with the outer gimbal axis. Therefore, it may be preferable to use other methods such as differential GPS signals to measure the aircraft attitude. Suppose an independent bias-free measurement $y_{o}(t)$ of the pitch angle is available as

$$
y_{o}=\theta .
$$

Since, the unknown bias $b$ is constant, the Eq. in (29) can be augmented with the equation: $\dot{b}=0$. This yields

$$
\begin{aligned}
\dot{x}_{o} & =A_{o} x_{o}+B_{o} q_{m} \\
y_{o} & =C_{o} x_{o}
\end{aligned}
$$

where

$$
x_{o}=\left[\begin{array}{l}
\theta \\
b
\end{array}\right] ; \quad A_{o}=\left[\begin{array}{cc}
0 & -1 \\
0 & 0
\end{array}\right] ; \quad B_{o}=\left[\begin{array}{l}
1 \\
0
\end{array}\right] ; \quad C_{o}=\left[\begin{array}{cc}
1 & 0
\end{array}\right] .
$$

Since $\left(C_{o}, A_{o}\right)$ is observable, an estimator can be constructed as

$$
\dot{\hat{x}}_{o}=A_{o} \hat{x}_{o}+B_{o} q_{m}+H\left(y_{o}-\hat{y}_{o}\right)
$$

where $H \in R^{2}$ is an observer gain (e.g., Kalman gain)

$$
H=\left[\begin{array}{ll}
h_{1} & h_{2}
\end{array}\right]^{T} .
$$

Using (30) and (32), we get the following estimation error dynamics

$$
\dot{\tilde{x}}_{o}=\left(A_{o}-H C_{o}\right) \tilde{x}_{o}
$$

where $\tilde{x}_{o}(t)=x_{o}(t)-\hat{x}_{o}(t)$ is the estimation error. Using (31) and (33) in (34), we have

$$
\left[\begin{array}{c}
\tilde{\tilde{\theta}} \\
\dot{\tilde{b}}
\end{array}\right]=\left[\begin{array}{cc}
-h_{1} & -1 \\
-h_{2} & 0
\end{array}\right]\left[\begin{array}{c}
\tilde{\theta} \\
\tilde{b}
\end{array}\right] \text {. }
$$


Since, the actual $\theta$ is measurable, the initial estimate of $\hat{\theta}(0)$ can be initialized to its true value, such that $\tilde{\theta}(0)=0$, while $\hat{b}(0)$ can be chosen arbitrarily.

The control law is defined as in (10), such that using the matching conditions in (12), the following closed-loop corrected-state equation is obtained:

$$
\dot{\bar{x}}=A_{m} \bar{x}+B_{m} r+B\left(\tilde{K}_{1}^{T} y+\tilde{K}_{2} r+\tilde{k}_{3}\right)-A_{m} \tilde{\beta}+\dot{\tilde{\beta}}
$$

where

$$
\tilde{\beta}=\left[\begin{array}{llll}
0 & 0 & 0 & \tilde{b}
\end{array}\right]^{T} .
$$

Substituting from (2) and (36) into (16), the following closed-loop error system is obtained:

$$
\dot{\hat{e}}=A_{m} \hat{e}+B\left(\tilde{K}_{1}^{T} y+\tilde{K}_{2} r+\tilde{k}_{3}\right)-A_{m} \tilde{\beta}+\dot{\tilde{\beta}} .
$$

Using (37) with (35), (38) can be expressed as

$$
\dot{\hat{e}}=A_{m} \hat{e}+B\left(\tilde{K}_{1}^{T} y+\tilde{K}_{2} r+\tilde{k}_{3}\right)-A_{m 4} \tilde{b}-\bar{h}_{2} \tilde{\theta}
$$

where $A_{m 4}$ represents the $4^{t h}$ column of $A_{m}$, and $\bar{h}_{2}=\left[\begin{array}{llll}0 & 0 & 0 & h_{2}\end{array}\right]^{T}$. Define a constant matrix $M \in R^{4 \times 2}$ such that

$$
M=\left[\begin{array}{ll}
\bar{h}_{2} & A_{m 4}
\end{array}\right] .
$$

Then (39) can be expressed as

$$
\dot{\hat{e}}=A_{m} \hat{e}+B\left(\tilde{K}_{1}^{T} y+\tilde{K}_{2} r+\tilde{k}_{3}\right)-M \tilde{x}_{o}
$$

The following result shows signal boundedness and asymptotic tracking using such a bias estimator.

Theorem 3: For the system given by (1), (2); the adaptive controller (10), the gain adaptation laws in (18), and the non-model-based observer in (32) guarantee that all the closed-loop signals including the adaptive gains are bounded and the tracking error $e(t) \rightarrow 0$ as $t \rightarrow \infty$.

Proof: Define

$$
V=\hat{e}^{T} P \hat{e}+\sum_{i}^{n} \tilde{K}_{1 i}^{T} \Gamma_{1}^{-1} \tilde{K}_{1 i}+\sum_{i}^{m_{r}} \tilde{K}_{2 i}^{T} \Gamma_{2}^{-1} \tilde{K}_{2 i}+\tilde{k}_{3}^{T} \Gamma_{3}^{-1} \tilde{k}_{3}+\tilde{x}_{o}^{T} P_{o} \tilde{x}_{o}
$$

where the subscript $i$ denotes the $i^{\text {th }}$ column of $\tilde{K}_{1}, \tilde{K}_{2}$; and $P_{o}=P_{o}^{T} \in R^{2 \times 2}$ is a positive definite solution to the Lyapunov inequality

$$
A_{o}^{T} P_{o}+P_{o} A_{o}<-Q_{o}
$$

for some positive definite matrix $Q_{o}=Q_{o}^{T} \in R^{2 \times 2}$. Differentiating (41) with respect to time, using (4), (40), (42), properties of matrix trace, and the gain update laws in (23), the following expression is obtained upon simplification:

$$
\begin{aligned}
\dot{V} & <-\hat{e}^{T} Q \hat{e}-2 \hat{e}^{T} P M \tilde{x}_{o}-\tilde{x}_{o}^{T} Q_{o} \tilde{x}_{o} \\
& =-z^{T} \bar{Q} z
\end{aligned}
$$

where $z \in R^{6}$ is defined as $z=\left[\begin{array}{ll}\hat{e} & \tilde{x}_{o}\end{array}\right]^{T}$, and $\bar{Q} \in R^{6 \times 6}$ is defined as

$$
\bar{Q}=\left[\begin{array}{cc}
Q & P M \\
(P M)^{T} & Q_{o}
\end{array}\right]
$$

Since $Q$ is positive definite, therefore $\dot{V}<0$ iff $Q_{o}-(P M)^{T} Q^{-1} P M$ is positive definite. $Q_{0}$ can be chosen such that $\bar{Q}$ is positive definite. The rest of the proof is similar to Theorem 2 . Therefore, all signals and estimates are bounded, and $\lim _{t \rightarrow \infty}\left(x-x_{m}\right)=0$. 


\section{V.B. Velocity Bias Observer}

Similar to the non-model-based observer designed to estimate the bias in pitch rate, an observer can be built to estimate the bias in true airspeed if reliable ground position and wind speed measurements are available. Consider that the available state measurement $(y=x+\beta)$ has an unknown constant bias in the true airspeed measurement, such that

$$
\beta=\left[\begin{array}{llll}
b & 0 & 0 & 0
\end{array}\right]^{T}
$$

where $b$ is an unknown constant. Thus, the measured airspeed $\left(v_{m}(t)\right)$ is expressed as

$$
v_{m}=v+b
$$

where $v(t)$ is the true airspeed. The true airspeed is related to the ground speed and the wind speed as

$$
v=v_{g}-v_{w}
$$

where $v_{g}(t)$ and $v_{w}(t)$ are the ground speed and the wind speed components along the flight path, respectively. Therefore using (44) in (43), we have

$$
v_{m}=v_{g}-v_{w}+b=\dot{x}_{p}-v_{w}+b
$$

where $x_{p}(t)$ is the ground position. Thus, the ground speed $\left(v_{g}=\dot{x}_{p}\right)$ is

$$
\dot{x}_{p}=-b+v_{m}+v_{w} .
$$

Suppose an independent bias-free measurement $y_{o}(t)$ of the ground position is available (e.g., using GPS signals) as

$$
y_{o}=x_{p}
$$

and in addition, the wind speed $v_{w}(t)$ measurement is available (e.g., from satellite data ${ }^{15}$ ). Then, (45) can be augmented with $\dot{b}=0$ to achieve an observable system similar to (30) and the unknown bias in true airspeed can be estimated. Closed-loop signal boundedness and asymptotic tracking can be shown in a manner similar to Section V.A.

Remark: The rate-gyro and the airspeed bias observers can be used simultaneously to estimate and accommodate the biases occurring simultaneously in the pitch-rate and airspeed measurements. Closed-loop signal boundedness and asymptotic tracking can be easily shown by extending the development in Sections V.A and V.B.

\section{Application Example- Large Transport Aircraft}

Simulation studies are performed on a fourth-order longitudinal dynamics model of a large transport aircraft in a wings-level cruise condition with known nominal trim conditions. In the presence of unknown sensor bias, the control objective is to track the response of a reference model to a reference input command superimposed with white noise (as shown in Figure 1). As in most MRAC schemes, the adaptation/estimation gains $\left(\Gamma^{\prime} s\right.$ and $\left.\eta\right)$ are selected by trial and error.

\section{VI.A. Dynamic Model}

The state variables for a fourth-order longitudinal dynamics model are: true airspeed $v(\mathrm{~m} / \mathrm{s})$, angle-of-attack $\alpha(\mathrm{deg})$, pitch angle $\theta(\mathrm{deg})$, and pitch rate $q(\mathrm{deg} / \mathrm{s})$. The actuators are elevator and engine throttle input, which produce control inputs $u_{e}(\mathrm{deg})$ and $u_{t}$, respectively. The elevator input $u_{e}$ represents the elevator position (deg) and the throttle input $u_{t}$ represents the thrust multiplied by a constant scale factor, and hence no units are used for $u_{t}$. Actuator dynamics are not considered. The linear time-invariant plant is described by (1), where the system matrices are

$$
A=\left[\begin{array}{cccc}
-0.0062 & -0.0815 & -0.1709 & -0.0026 \\
-0.0344 & -0.5717 & 0 & 1.0050 \\
0 & 0 & 0 & 1.0000 \\
0.0115 & -1.0490 & 0 & -0.6803
\end{array}\right], B=\left[\begin{array}{cc}
0 & 1.3287 \\
-11.4027 & -0.0401 \\
0 & 0 \\
-44.5192 & 0.8824
\end{array}\right]
$$


The state is $x(t)=\left[\begin{array}{llll}v & \alpha & \theta & q\end{array}\right]^{T}$, and the control input is $u(t)=\left[\begin{array}{ll}u_{e} & u_{t}\end{array}\right]^{T}$.

(For other flight conditions such as coordinated turns, the model would be different).

\section{VI.B. Reference Model}

The reference model is chosen as:

$$
A_{m}=A+B K_{1}^{T}, B_{m}=B K_{2}
$$

where $K_{1}$ is the LQR gain designed to obtain satisfactory closed-loop response. The gain $K_{2}=I_{2}$, so that $B_{m}=B$. In practice, $K_{2}$ is chosen to provide proper scaling of the reference input $r(t)$.

\section{VI.C. Simulation Cases}

To test the performance of various adaptive control laws presented in this paper, simulations are performed for the following four cases in the presence of unknown sensor bias:

- Case 1: Standard MRAC with biased measurements (Section II)

- Case 2: Feedback-based bias estimation with MRAC (Section III)

- Case 3: Use of asymptotic bias estimator with MRAC (Section IV)

- Case 4: Use of non-model-based observers with MRAC (Section V)

In each of these cases, it is assumed that the unknown bias appears at $t=0$.

Case 1: The unknown constant bias in the state measurement is arbitrarily chosen as

$$
\beta=\left[\begin{array}{llll}
5 & 2 & -1 & 10
\end{array}\right]^{T} .
$$

The units of the components of $\beta$, which represent the biases in $(v, \alpha, \theta, q)$, are $\mathrm{m} / \mathrm{s}, \mathrm{deg}$, deg, and $\mathrm{deg} / \mathrm{s}$ respectively. Using the biased measurements, the standard MRAC control law (5) of Section II is implemented with the adaptive gains $\hat{K}_{1}$ and $\hat{K}_{2}$ initialized to half of their true values, and the adaptation gains are chosen arbitrarily, so that

$$
\hat{K}_{1}(0)=0.5 K_{1}, \hat{K}_{2}(0)=0.5 K_{2}, \Gamma_{1}=0.005 I_{4}, \Gamma_{2}=0.005 I_{2} .
$$

The plant and reference model states, tracking errors, and control input are shown in Figures 2, 3, and 4, respectively. As stated in Section II, the closed-loop signal boundedness or bounded tracking error cannot be proved. However, in this example, the tracking errors appear to approach some non-zero constant values.

Case 2: For the sensor bias as in (46), the control law in (10) is implemented where the bias estimate is generated using the bias estimation law in (19). The gain $\hat{k}_{3}$ is initialized to zero with adaptation rate $\Gamma_{3}=0.005 I_{2}$, while other gains are chosen as in (47). The plant and reference model states, tracking errors, control input, and bias estimates are shown in Figures 5, 6, 7, and 8, respectively. Except for velocity, the controller and the bias estimator effectively compensate for the sensor bias, and achieve an acceptable tracking performance. The bias estimates converge to values that are not necessarily the true values. A better tracking performance and bias estimation may be possible if the persistent excitation (PE) condition is satisfied.

Case 3: For the same sensor bias as in (46), the adaptive control law (10) of Section IV is implemented. The adaptive gain initialization and adaptation gains are chosen same as in Cases 1 and 2. The matrix $A_{\beta}$ of the asymptotic bias estimator in (22) is arbitrarily chosen as

$$
A_{\beta}=1.1 A_{m} .
$$

The bias estimate converges to its true value as shown in Figure 12. The plant and reference model states, tracking errors, and control input are shown in Figures 9, 10, and 11, respectively. The tracking errors converge to zero asymptotically.

Case 4: The bias vector considered in this case is different compared to the other cases because in order to use the non-model-based observer developed in Section V, it is assumed that the bias exists only in the 
airspeed and pitch-rate measurements, while reliable bias-free measurements of angle-of-attack and pitch angle are available. Therefore,

$$
\beta=\left[\begin{array}{llll}
25 & 0 & 0 & 10
\end{array}\right]^{T} .
$$

The adaptive gain initialization and adaptation gains are chosen same as in Case 2. The wind speed is assumed to be known as $v_{w}=10(\mathrm{~m} / \mathrm{s})$. The observer gain is chosen so as to obtain a fast convergence (within $1.0 \mathrm{sec}$ ), as indicated by the bias estimates convergence in Figure 16. The plant and reference model states, tracking errors, and control input are shown in Figures 13, 14, and 15, respectively. The tracking errors converge to zero asymptotically.

However, if the standard MRAC control law in (5) is implemented with a bias in airspeed and pitch rate feedback without compensating for the sensor bias via an observer, then there is a large offset in the airspeed, angle-of-attack and pitch angle as indicated by the plant response shown in Figure 17.

\section{VI.D. Discussion}

The steady-state tracking errors improve as the design progresses from Case 1 to Case 3 (cf. Figures 3, 6, 10). Also note the improvements in the steady-state condition of the airplane as the design progresses from Case 1 to Case 3 (cf. Figures 2, 5, 9). In Case 1, using just the standard MRAC control law, the airplane trims $5(\mathrm{~m} / \mathrm{s})$ too slow (consistent with the $5(\mathrm{~m} / \mathrm{s})$ too high sensor measurement) and at a flight-path angle $(\gamma=\theta-\alpha)$ of about -4 (deg) (descending steeper than a normal landing approach). In Case 2, with the addition of feedback-based bias estimation to the MRAC control law, the airplane flight-path angle is partially corrected to about -1 (deg) while the speed error is not affected. In Case 3, with an asymptotic bias estimator, both the flight-path angle and the speed are fully corrected.

In Case 4, the bias only exists in airspeed and pitch-rate measurements. The non-model-based observer developed in Section V combined with MRAC is able to achieve asymptotic tracking (Figure 13). However, if a standard MRAC approach is used, then the steady-state response has large offsets (Figure 17). In this case the aircraft has a glide path angle of about -9 degrees. The aircraft is rapidly coming down while the reference model is not. This shows that when using standard MRAC, the sensor bias has adverse effects on both the steady-state tracking performance and the steady-state flight condition of the airplane.

\section{Concluding Remarks}

This paper addressed the problem of accommodating unknown sensor bias in control of uncertain systems. Such a bias can have serious detrimental effects on closed-loop stability and performance. In particular, the standard model reference adaptive control (MRAC) law for state tracking cannot guarantee signal boundedness or boundedness of the tracking error if biased sensor data is used to synthesize the control input. Therefore, a modified MRAC control law was developed, which incorporates sensor bias estimation along with gain adaptation, and was shown to guarantee signal boundedness and bounded state tracking error. For a case wherein a separate asymptotically convergent bias estimator is available, an MRAC control law was developed and was shown to provide signal boundedness and asymptotic tracking. Such asymptotically convergent estimators can be designed if additional independent sensor measurements are available. An example of a bias estimator was presented for longitudinal aircraft dynamics with constant biases in the pitch rate gyro and the airspeed measurements. It was also shown that the bias can be estimated and used in conjunction with MRAC to guarantee asymptotic state tracking and closed-loop stability. Numerical examples were presented to demonstrate the proposed schemes.

The examples presented in this paper consisted of longitudinal dynamics in a wings-level cruise flight. Other flight conditions such as coordinated turns should be considered in future work using a six degree-offreedom model. Application of the method to a full nonlinear model should also be investigated. Future work should also focus on extending the results to a broad class of sensor faults (including unknown scale factor and time-varying bias), and on methods for constructing bias estimators using data fusion for all available multiple redundant sensors. In addition, the methods need to be extended to address actuator faults that occur simultaneously with sensor faults, in order to effectively prevent loss of control in aircraft. 


\section{Acknowledgment}

This research was supported in part by an appointment to the NASA Postdoctoral Program at Langley Research Center, administered by Oak Ridge Associated Universities through a contract with NASA.

\section{References}

${ }^{1}$ Alea, B.J.M., Bellamyb, L.J., Cooperc, J.,Ababeid, D., Kurowickad,D., Moralesd, O., and Spougee, J.: "Analysis of the crash of TK 1951 using CATS ", Reliability Engineering 85 System Safety, Volume 95, Issue 5, May 2010, pp. 469-477

${ }^{2}$ Anon: "Qantas Airbus A330 accident Media Conference", Press release Australian Transport Safety Bureau, 14 October 2008.

${ }^{3}$ Anon: "Airbus gives new warning on speed sensors", (Associated Press article),

http://www.cbc.ca/world/story/2010/12/21/airbus-sensor-warning.html, Canadian Broadcasting Corp. (CBC) News, December 21,2010 .

${ }^{4}$ Tao, G. (2003)Adaptive Control Design and Analysis, N.J.: John Wiley \& Sons.

${ }^{5}$ Tao, G., Joshi, S. M., and Ma, X. (2001), “Adaptive State Feedback and Tracking Control of Systems with Actuator Failures", IEEE Trans. on Automatic Control, vol. 46, No. 1, pp. 78-95.

${ }^{6}$ Tao, G., Chen, S. H., Tang, X. D., Joshi, S. M., "Adaptive Control of Systems with Actuator Failures", London: SpringerVerlag, 2004.

${ }^{7}$ Tao, G., and Joshi, S. M., "Direct Adaptive Control of Systems with Actuator Failures: State of the Art and Continuing Challenges", Proc. 2008 AIAA Guidance, Navigation, and Control Conference, Honolulu, HI, Aug 18-21.

${ }^{8}$ Bevly, David M., "Velocity Sensor for Correcting Inertial Sensor Errors on Ground Vehicles", J. Dyn. Sys., Meas., Control, vol. 126, Issue 2, June 2004, pp. 255-264.

${ }^{9}$ Vemuri, A.T.; "Sensor bias fault diagnosis in a class of nonlinear systems", IEEE Trans. Auto. Contr., vol. 46, Issue 6, June 2001, pp. 949 - 954.

${ }^{10}$ Healey, A.J., An, E.P., and Marco, D.B., "Online compensation of heading sensor bias for low cost AUVs", Proc. 1998 Workshop on Autonomous Underwater Vehicles, 20-21 Aug, 1998, pp. 35-42.

${ }^{11}$ Chandrasekar, J., and Bernstein, D.S., "Setpoint tracking with actuator offset and sensor bias - Probing the limits of integral control", IEEE Control Systems Magazine, Feb. 2007, pp. 61 - 68.

${ }^{12}$ Tao, G. and Burkholder, J. O., "Adaptive detection of sensor uncertainties and failures," Proceedings of 2009 AIAA Guidance, Navigation and Control Conference, Paper AIAA-2009-5889, Chicago, IL, August 2009.

${ }^{13} \mathrm{Li}, \mathrm{S}$., and Tao, G.: "Output Feedback MIMO MRAC Schemes with Sensor Uncertainty Compensation", 2010 American Control Conference, Baltimore, MD, USA, June 30-July 02, 2010, pp. 3229-3234.

${ }^{14}$ Sun, Z., and Joshi, S. M., "An Indirect Adaptive Control Scheme in the Presence of Actuator and Sensor Failures", 2009 AIAA Guidance, Navigation, and Control Conference, Chicago, Aug 10-13.

${ }^{15}$ Monaldo, F.M., Thompson, D.R., Pichel, W.G., and Clemente-Colon, P., "Application and extension of a quasioperational approach to wind speed measurement from spaceborne synthetic aperture radar", 2004 Geoscience and Remote Sensing Symposium, Vol. 1, 2004, pp. 144-147.

Figure 1. Reference command for all cases
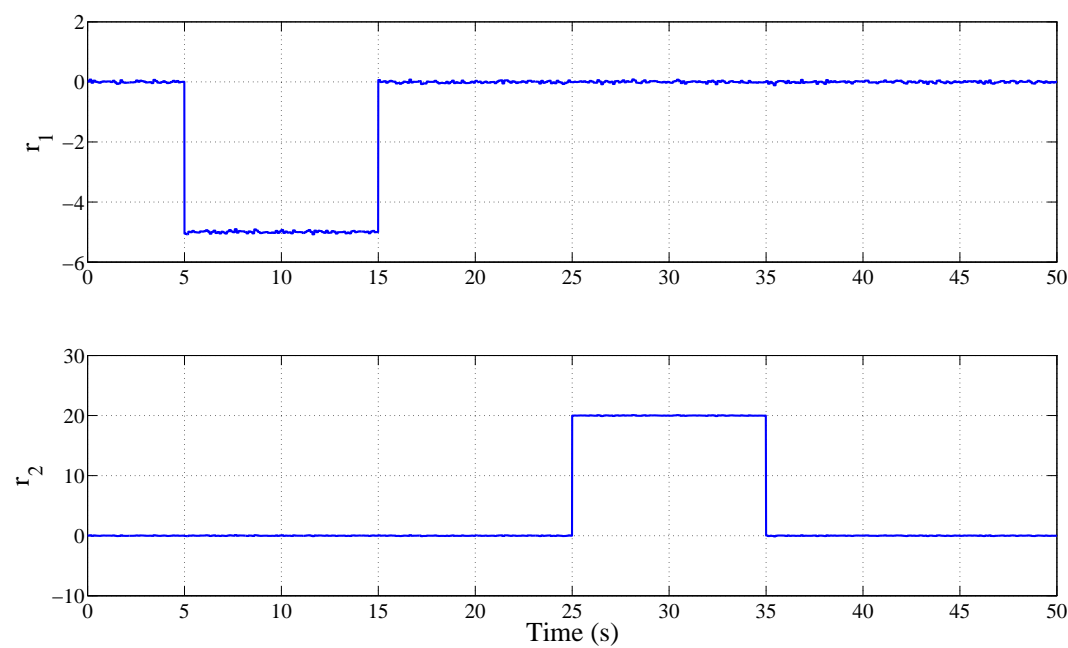

11 of 18 
Figure 2. Case 1: Plant and reference model states
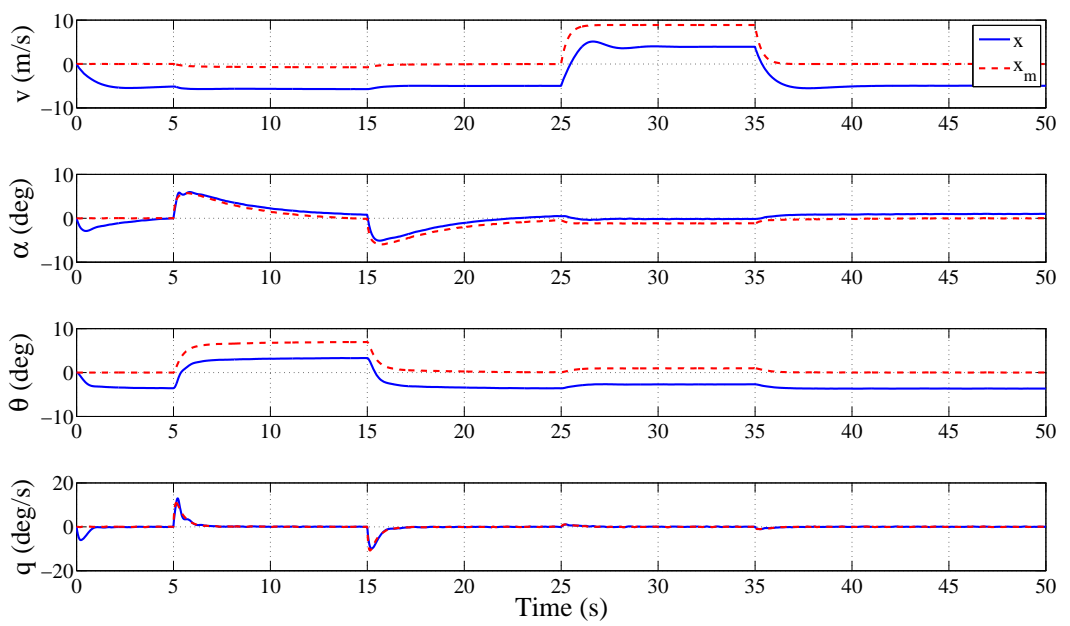

Figure 3. Case 1: State tracking error
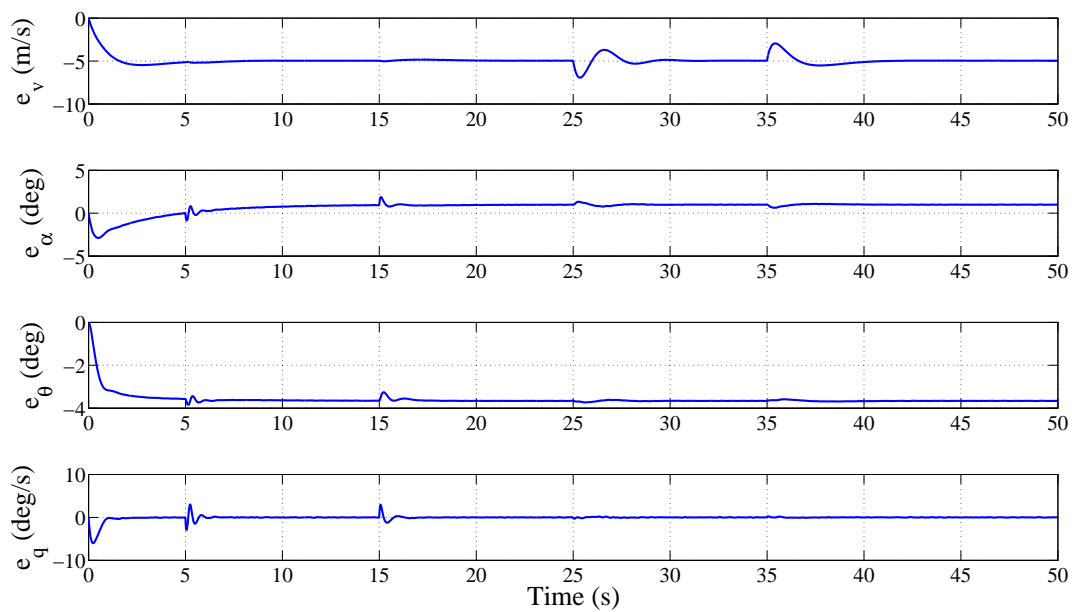

12 of 18 
Figure 4. Case 1: Control input
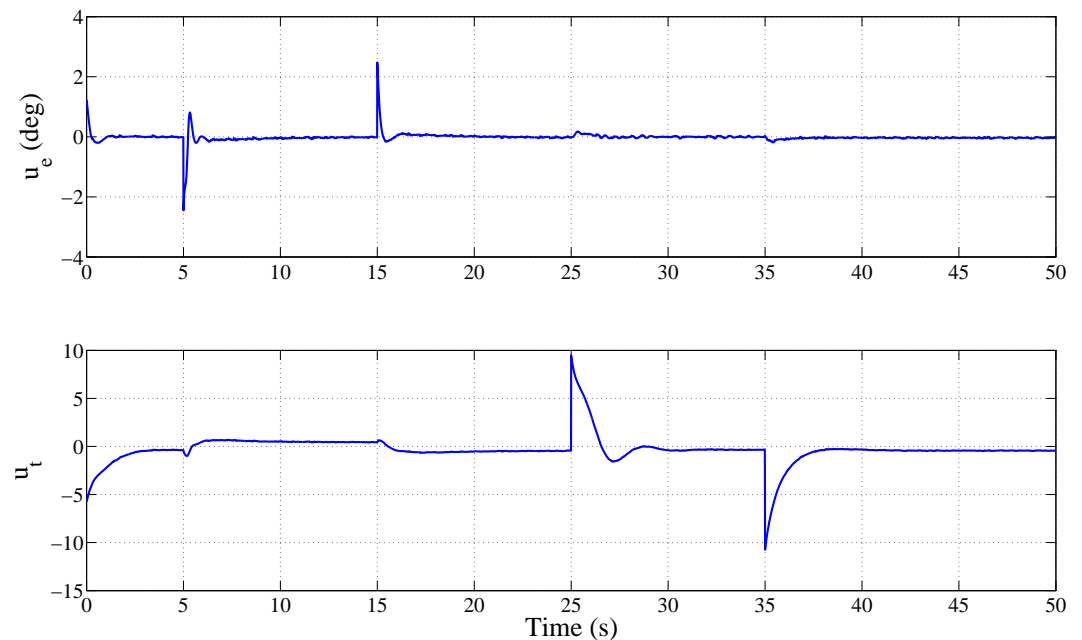

Figure 5. Case 2: Plant and reference model states
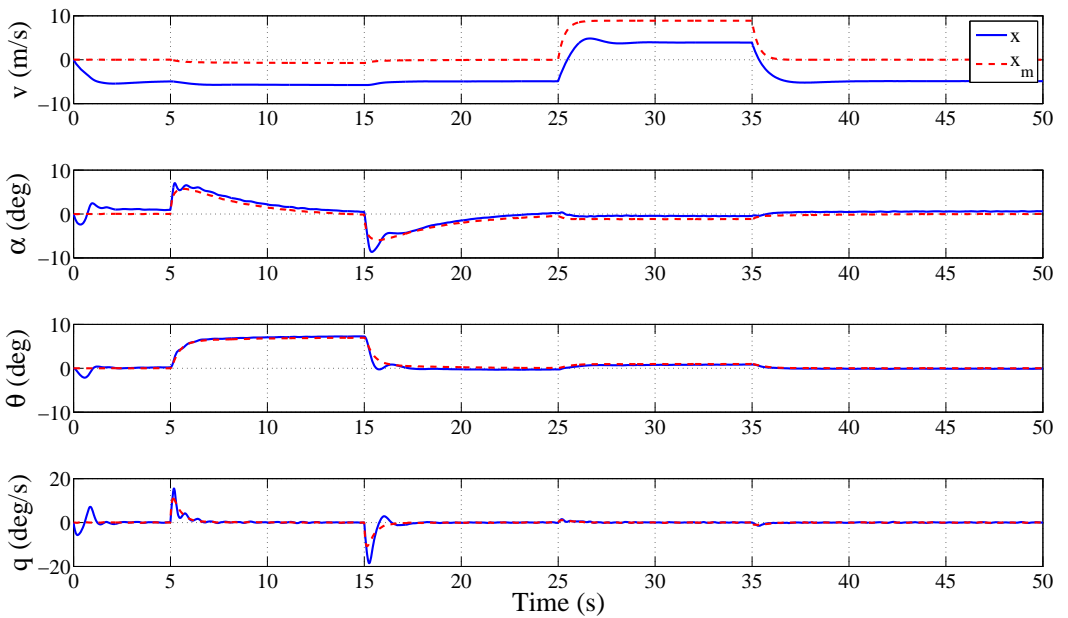
Figure 6. Case 2: State tracking error
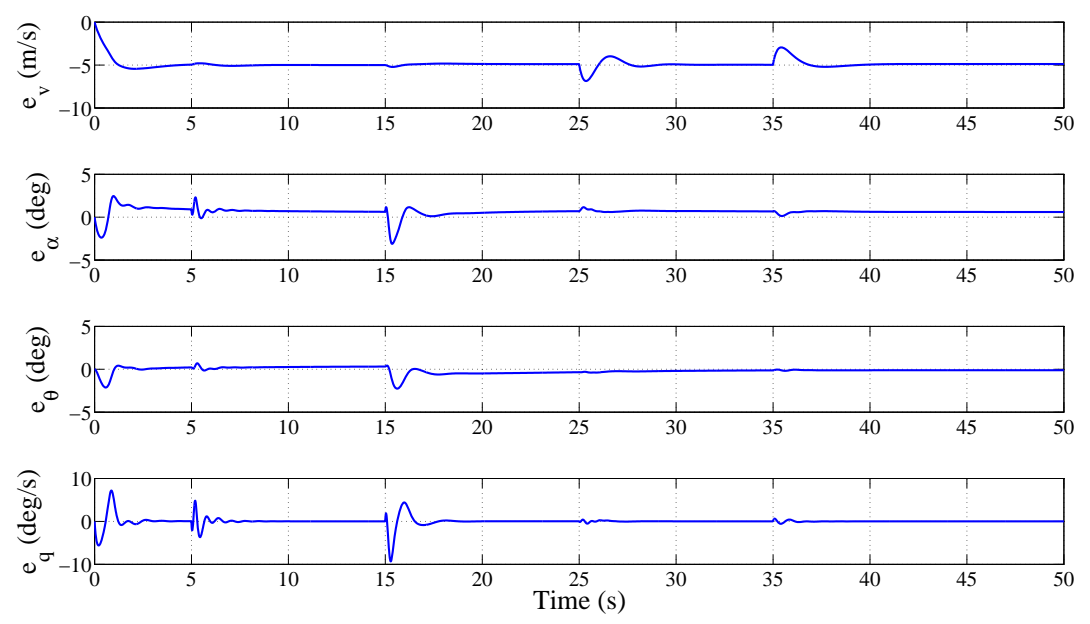

Figure 7. Case 2: Control input
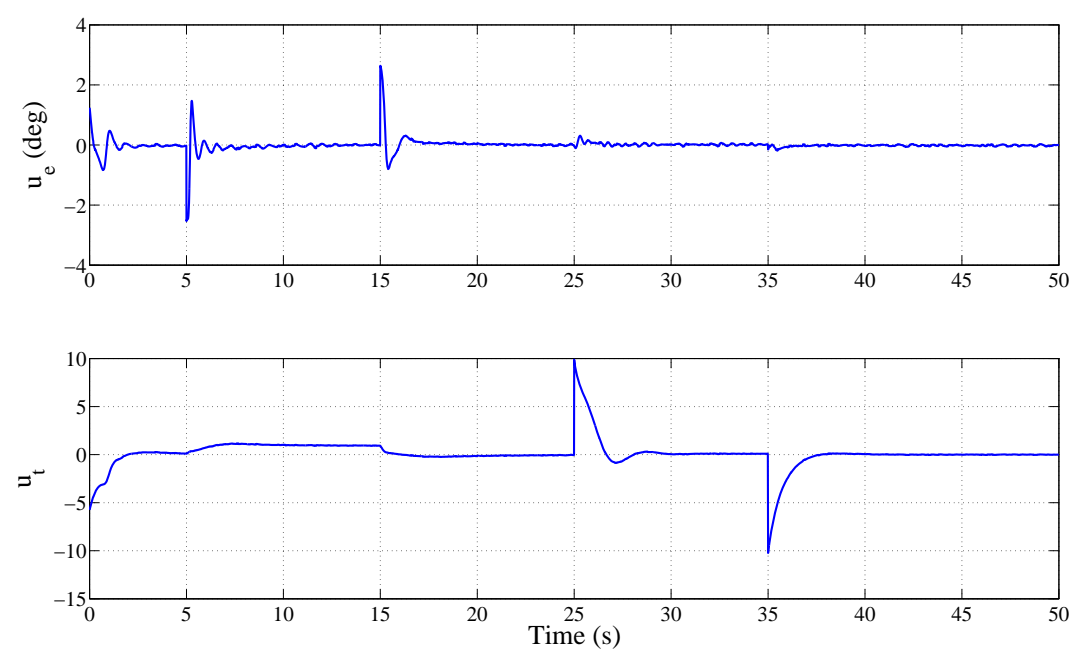

Figure 8. Case 2: Bias estimate

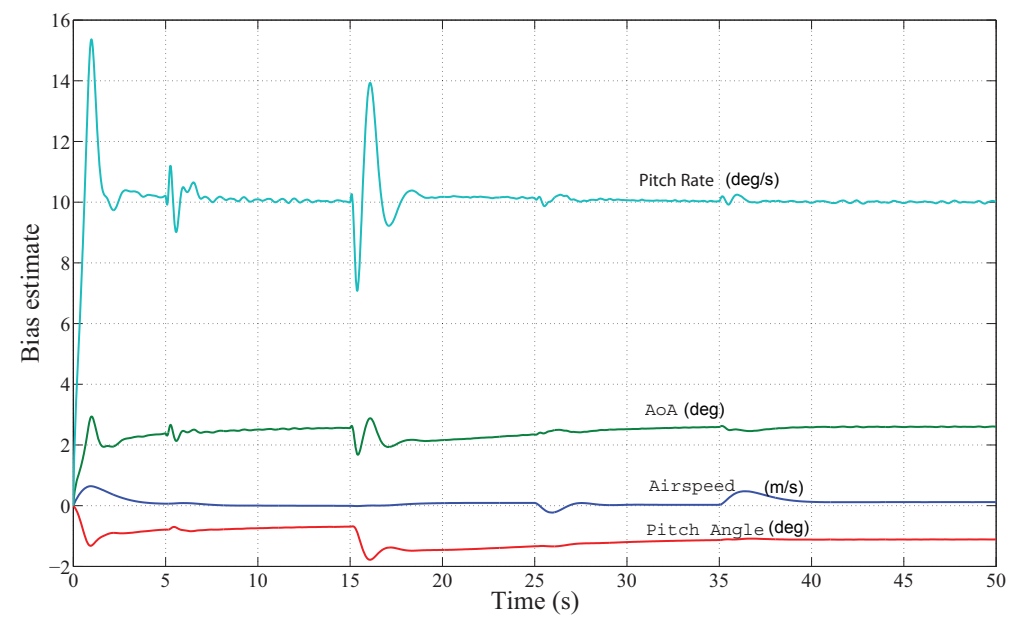

14 of 18 
Figure 9. Case 3: Plant and reference model states
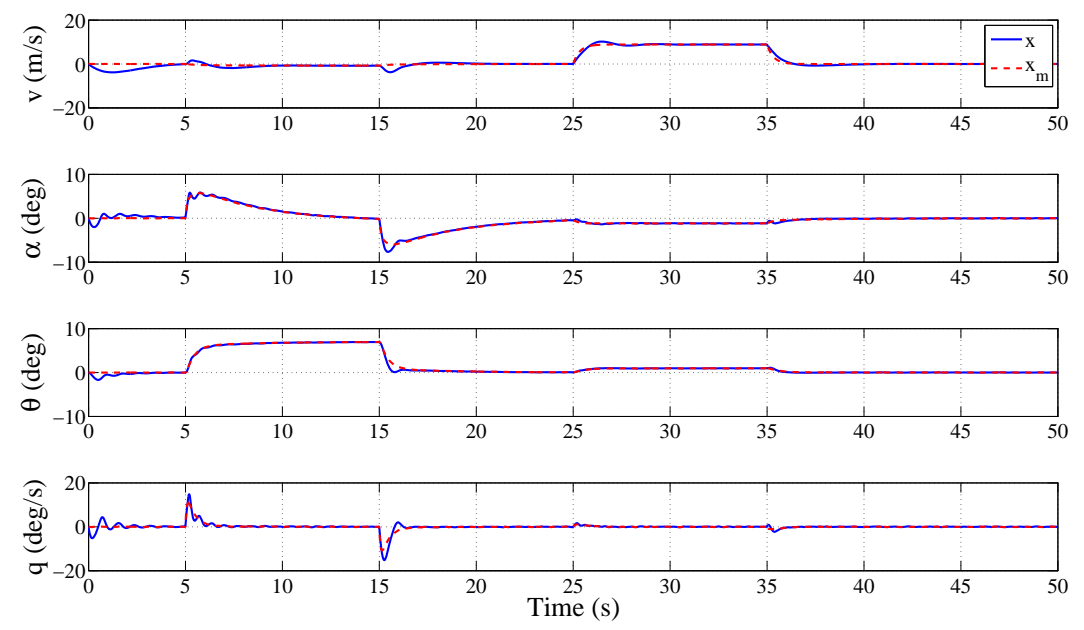

Figure 10. Case 3: State tracking error
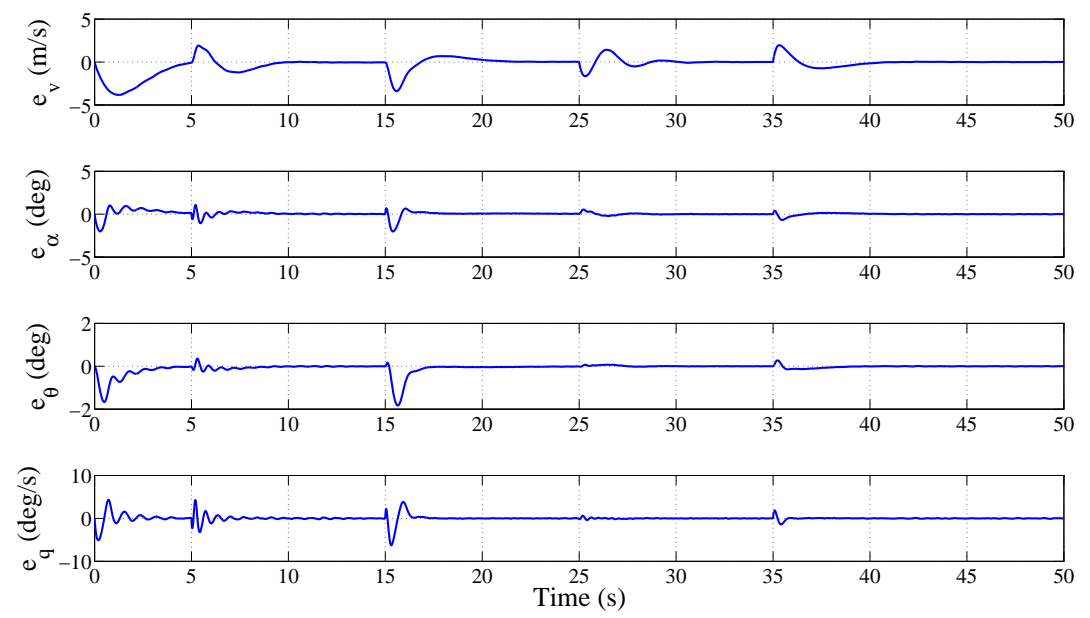
Figure 11. Case 3: Control input
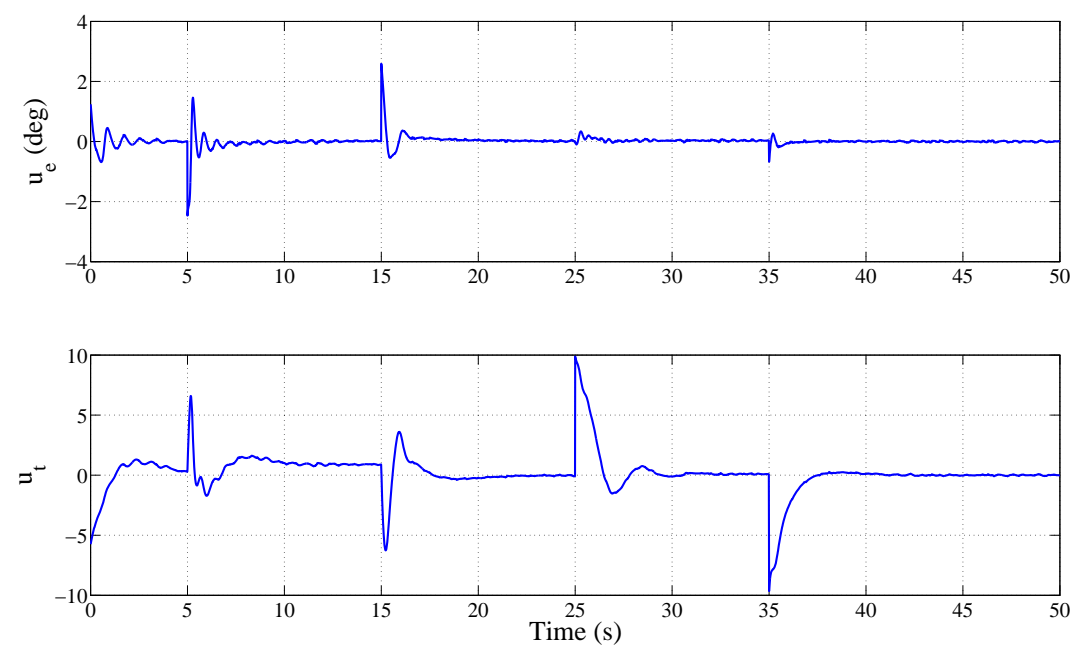

Figure 12. Case 3: Bias estimate

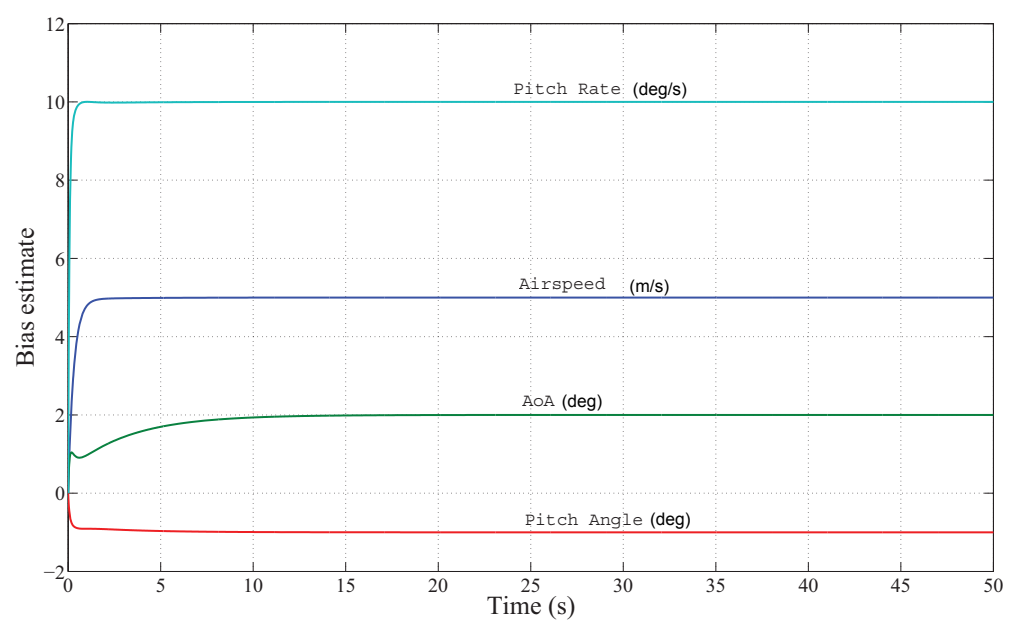

Figure 13. Case 4: Plant and reference model states
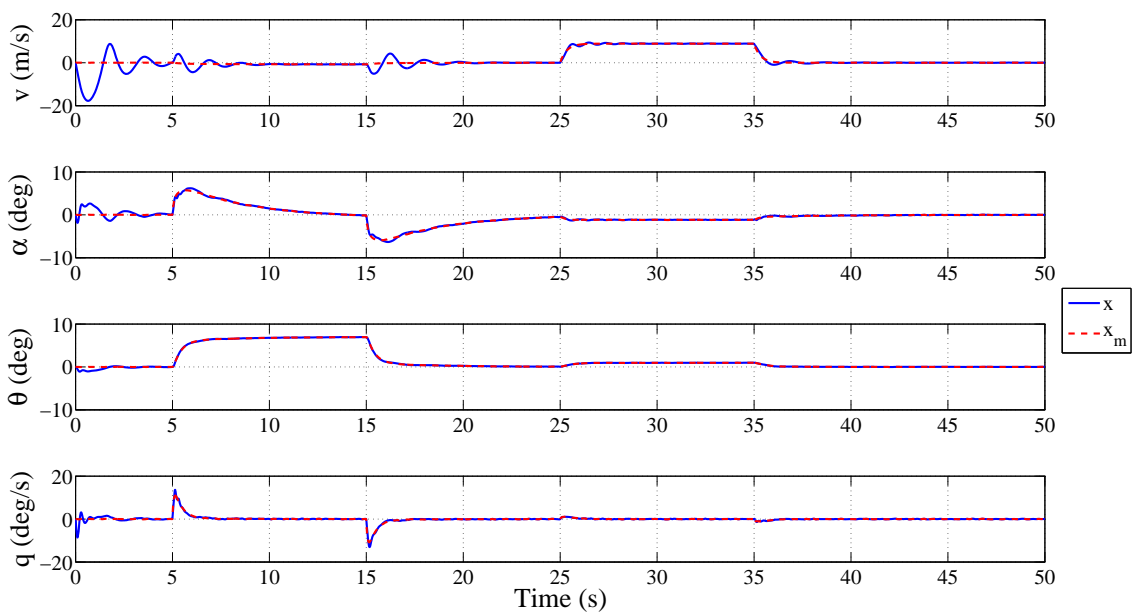

16 of 18 
Figure 14. Case 4: State tracking error
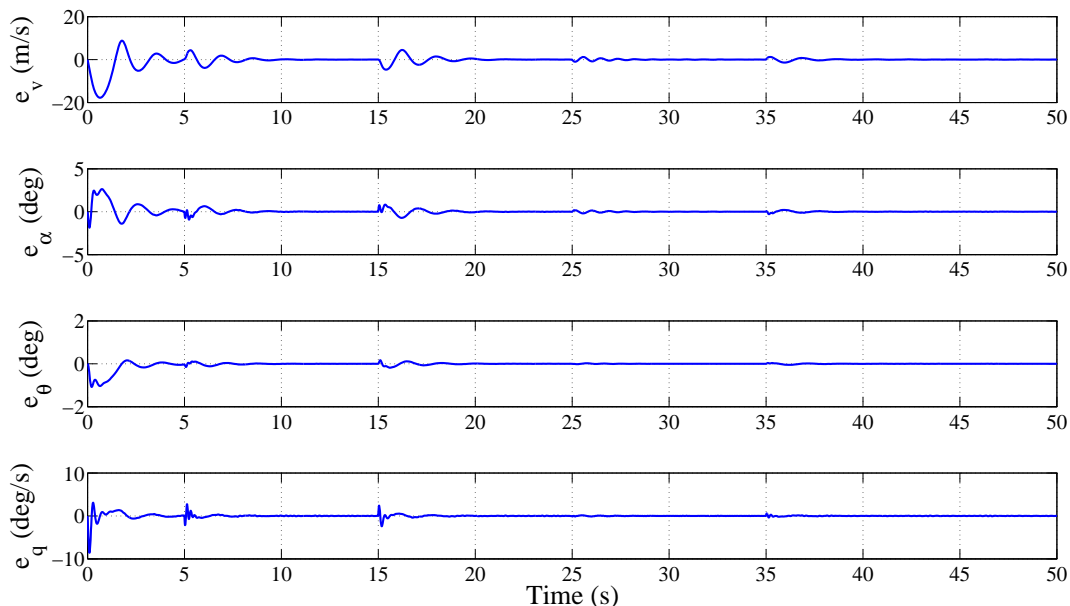

Figure 15. Case 4: Control input
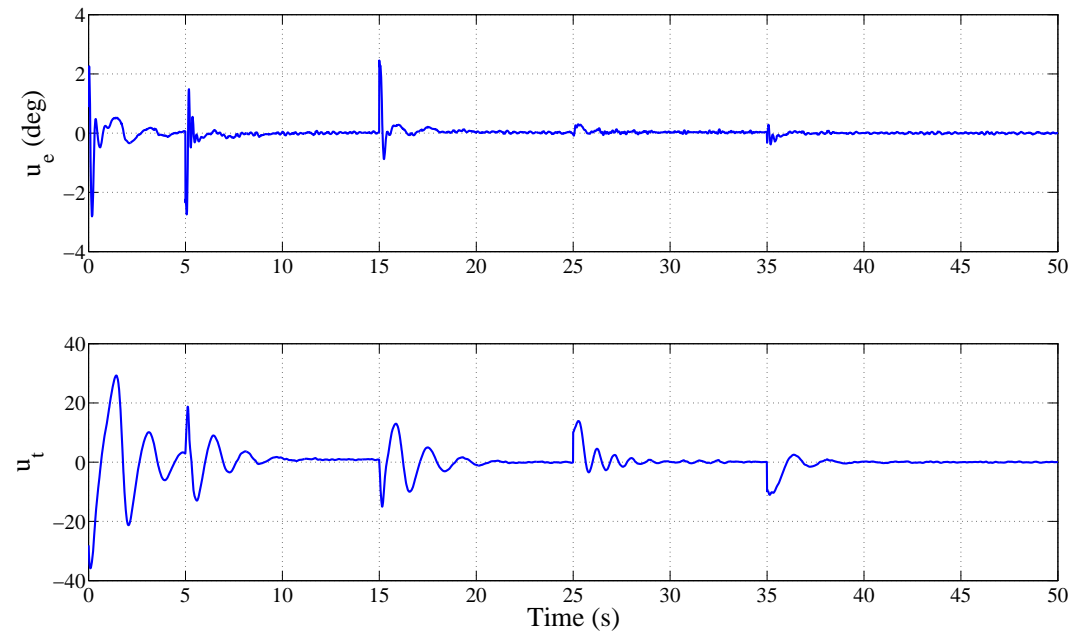

Figure 16. Case 4: Bias estimate

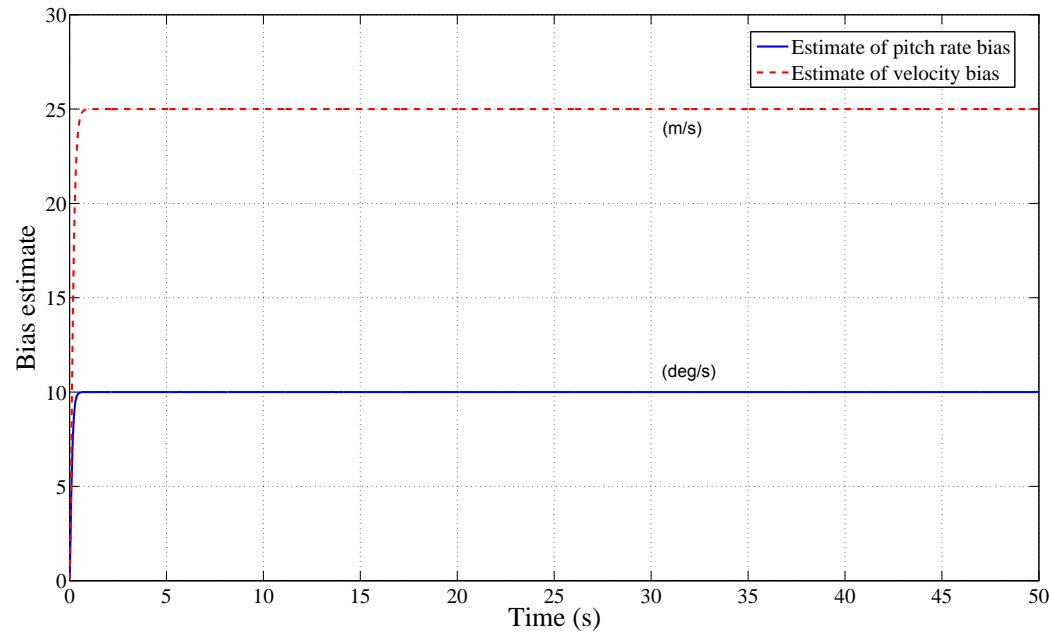

17 of 18 
Figure 17. Case 4: Plant and reference model states without using a non-model-based observer
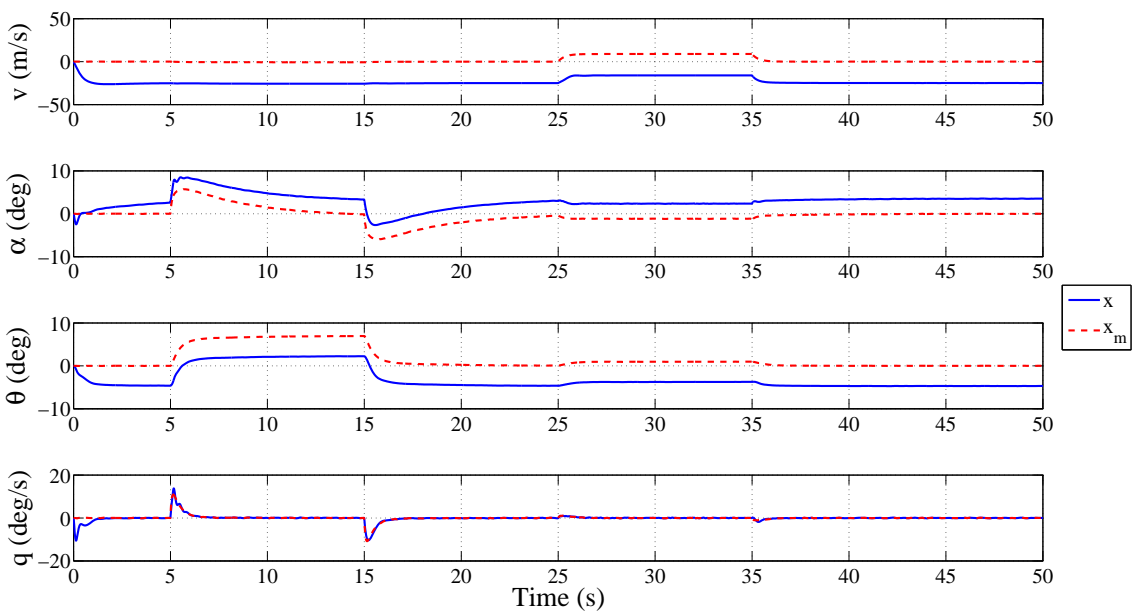

18 of 18 